\title{
A pendulum with a broken cylindrical symmetry: normal modes and beats
}

\author{
Gilles Dolfo and Jacques Vigué \\ Laboratoire Collisions Agrégats Réactivité-IRSAMC \\ Université de Toulouse-UPS and CNRS UMR 5589 \\ 118, Route de Narbonne, 31062 Toulouse Cedex, France \\ E-mail: jacques.vigue@irsamc.ups-tlse.fr
}

\begin{abstract}
.
A pendulum, which has the cylindrical symmetry around its suspension wire, has two degenerate normal modes. We break this symmetry by putting a small magnet in the pendulum body and by running the pendulum in the presence of an inhomogeneous magnetic field. The force due to the magnetic field gradient modifies the equilibrium position and the spatial derivatives of this force define the normal modes of the pendulum with two different oscillation frequencies. We describe this system theoretically and we test these results by a series of experiments. This system provides a classical analogue of the degenerate perturbation theory in quantum mechanics.
\end{abstract}

PACS numbers: 


\section{Introduction}

Since the discovery of the isochronism of small oscillations by Galileo Galilei, the pendulum has been used for an extraordinary variety of physical and technical applications $[1,2,3]$. In the present paper, we analyze the normal modes of a pendulum with a slightly broken cylindrical symmetry. When this symmetry is perfect, the normal modes are degenerate: the pendulum can oscillate in any direction with the same frequency and any combination of two orthogonal oscillations, which represents an elliptic oscillation, is also a solution of Newton's equations of motion. If a perturbation breaks the cylindrical symmetry, the frequency degeneracy of the normal modes is lifted. If the perturbation is conserved by time reversal, the normal modes correspond to two orthogonal directions of oscillation: this behavior is illustrated by the Blackburn pendulum also called the $\mathrm{Y}$-suspended pendulum $[4,5]$ and by the experiment described in the present paper. If the perturbation changes sign by time reversal, the normal modes are circular oscillations in opposite sense of rotation as illustrated by the Foucault pendulum [6]: for this pendulum, the mode degeneracy is lifted by the Coriolis force and this pendulum provides a classical effect of a geometric phase [7,8].

Finally, the lifting of an eigenstate degeneracy is well known in perturbation theory in quantum mechanics and the present study provides a perfect analogue in classical mechanics. In both cases, starting from a degenerate situation, the perturbation defines the new eigenstates or the new normal modes which are obtained by the diagonalization of the perturbation matrix expressed in the initial basis describing the degenerate normal modes.

We have built a pendulum made of sphere supported a piano string. This suspension makes that the pendulum is a good example of $2 \mathrm{D}$ quasi-harmonic oscillator (the usual suspension by a spring used in clocks or the use of knives limit the pendulum motion to in-plane oscillations and the pendulum is then described as a 1D quasiharmonic oscillator [1]). A small magnet was placed near the sphere center in order to excite the pendulum oscillation in a controlled manner, thanks to the force due to a magnetic field gradient. If the laboratory magnetic field is inhomogeneous, the pendulum cylindrical symmetry is broken. The pendulum equilibrium position is then displaced, proportionally to the first derivatives of the field, and the frequency degeneracy of the normal modes is lifted, the new normal modes corresponding to linear oscillations. The rigidity with respect to torsion of the piano string is large so that the rotation of the magnet in the horizontal plane is negligible.

We develop the theory of this effect in the general case and in the practical case of

the magnetic field produced by a magnetic dipole. If the two normal modes are excited simultaneously with equal oscillation amplitudes, one observes a beat pattern, with the oscillation varying from linear in one direction to linear in the perpendicular direction, with intermediate periods of elliptic and even circular oscillations. All the calculations are feasible by undergraduate students and the experiment is easy to build.

This paper is organized as follows: we first develop the theory of a pendulum 
carrying a magnet in the presence of an inhomogeneous magnetic field; we then express the magnetic field of a dipole in cartesian coordinates; its first and second spatial derivatives, which are presented in an appendix, are used to calculate the magnetic field effect on the pendulum motion ; we describe our experimental set-up and some experimental tests of the theory. We present some concluding remarks.

\section{Theory}

\subsection{The pendulum}

We consider a pendulum having a perfect cylindrical symmetry around the vertical axis except that the pendulum body carries a small magnet, of magnetic moment $\mathbf{m}_{P}$, which interacts with the laboratory magnetic field $\mathbf{B}$. We consider only small amplitude oscillations and we neglect the torsion of the suspension wire so that the direction of the magnetic moment $\mathbf{m}_{P}$ is fixed in space.

\subsection{Newton's equations in zero magnetic field}

We describe the pendulum as a point mass $M$ at the end of a wire of length $l$. The equilibrium position chosen as the origin $O$ of the coordinate system with the $\mathbf{x}$ and $\mathbf{y}$ axes in the horizontal plane. The $\mathbf{z}$-axis is vertical, oriented upward. Because of the wire, the mass $M$ is on the sphere of radius $l$ centered at $x=y=0, z=l$ and, neglecting fourth order terms in $x, y$, its altitude is $z \approx\left(x^{2}+y^{2}\right) /(2 l)$. The gravitational potential energy is given by

$$
E_{g}=M g z \approx M g \frac{x^{2}+y^{2}}{2 l},
$$

where $g$ is the local acceleration of gravity. From $E_{g}$, we deduce the restoring force $\mathbf{F}_{g}=-\nabla E_{g}$ and Newton's equations of motion

$$
\begin{aligned}
& M \frac{d^{2} x}{d t^{2}} \approx-k_{g} x, \\
& M \frac{d^{2} y}{d t^{2}} \approx-k_{g} y .
\end{aligned}
$$

We note $k_{g}=M g / l$ the gravitational restoring force constant. The motion is harmonic

in the $\mathbf{x}$ and $\mathbf{y}$ directions with the same angular frequency $\omega=\sqrt{k_{g} / M}=\sqrt{g / l}$. The two normal modes, corresponding to oscillations along the $\mathbf{x}$ and $\mathbf{y}$ axes, are degenerate and any other choice of orthogonal axes is equivalent. The general solution of Newton's equations is

$$
\begin{aligned}
& x=a_{x} \cos \left(\omega t+\varphi_{x}\right), \\
& y=a_{y} \cos \left(\omega t+\varphi_{y}\right) .
\end{aligned}
$$

These equations describe any linear or elliptic harmonic oscillation of angular frequency $\omega$. 


\subsection{Newton's equations in the presence of a magnetic field}

If the magnetic moment $\mathbf{m}_{P}$ interacts with the external magnetic field $\mathbf{B}$, there is a supplementary potential energy $E_{m}$

$$
E_{m}=-\mathbf{m}_{P} \cdot \mathbf{B}=-\sum_{i=1}^{3} m_{P, i} B_{i}
$$

where the indices $i=1,2,3$ are used to label the $x, y, z$ coordinates and the cartesian components of the vectors. The magnetic force $\mathbf{F}_{m}$ exerted on the magnet is given by $\mathbf{F}_{m}=-\nabla E_{m}$ whose components are given by

$$
F_{m, j}=\sum_{i=1}^{3} m_{P, i} \frac{\partial B_{i}}{\partial x_{j}}
$$

If the magnetic force is very small with respect to the weight $M \mathbf{g}$, the sole effect of the vertical component of the force is to slightly correct the weight, a negligible effect. The horizontal components of the magnetic force displace the equilibrium position to the point where the total force vanishes. The coordinates $x_{e, j}$ of the new equilibrium position are

$$
x_{e, j}=\sum_{i=1}^{3} \frac{m_{P, i}}{k_{g}} \frac{\partial B_{i}}{\partial x_{j}} \text { for } j=1,2
$$

The pendulum oscillation is sensitive to the second derivatives of the potential energy calculated in the $x-y$ plane at the new equilibrium position. We consider small horizontal displacements $\epsilon_{i}$ from the new equilibrium position and we limit the total potential energy $E_{\text {pot }}=E_{g}+E_{m}$ to its second order Taylor expansion

$$
\begin{aligned}
& E_{\text {pot }} \quad=E_{\text {pot }}\left(x_{e}, y_{e}\right)+\frac{1}{2} \sum_{i, j}\left[k_{g} \delta_{i j}+k_{m, i, j}\right] \epsilon_{i} \epsilon_{j} \\
& \text { with } k_{m, i, j}=k_{m, j, i}=\frac{\partial^{2} E_{m}}{\partial x_{i} \partial x_{j}}=-\sum_{k=1}^{3} m_{P, k} \frac{\partial^{2} B_{k}}{\partial x_{i} \partial x_{j}}
\end{aligned}
$$

where $\delta_{i j}$ is the Kronecker symbol. We derive Newton's equations of motion

$$
M \frac{d^{2}}{d t^{2}}\left[\begin{array}{c}
\epsilon_{x} \\
\epsilon_{y}
\end{array}\right]=-\left[\begin{array}{cc}
k_{g}+k_{m, x, x} & k_{m, x, y} \\
k_{m, x, y} & k_{g}+k_{m, y, y}
\end{array}\right]\left[\begin{array}{c}
\epsilon_{x} \\
\epsilon_{y}
\end{array}\right] .
$$

As this matrix is symmetric, it can be diagonalized by a rotation of the coordinate system to new axes $\mathbf{u}$ and $\mathbf{v}$. Newton's equations are then uncoupled

$$
M \frac{d^{2}}{d t^{2}}\left[\begin{array}{l}
u \\
v
\end{array}\right]=-\left[\begin{array}{cc}
k_{g}+k_{m, u, u} & 0 \\
0 & k_{g}+k_{m, v, v}
\end{array}\right]\left[\begin{array}{l}
u \\
v
\end{array}\right] .
$$

Provided that $\left(k_{g}+k_{m, u, u}\right)$ and $\left(k_{g}+k_{m, v, v}\right)$ are both positive (in the opposite case, the new equilibrium position is unstable), the normal modes describe harmonic oscillations along the new axes with the angular frequencies

$$
\omega_{u}=\sqrt{\frac{k_{g}+k_{m, u, u}}{M}} \approx \omega\left(1+\frac{k_{m, u, u}}{2 k_{g}}\right)
$$


A pendulum with a broken cylindrical symmetry: normal modes and beats

$$
\omega_{v}=\sqrt{\frac{k_{g}+k_{m, v, v}}{M}} \approx \omega\left(1+\frac{k_{m, v, v}}{2 k_{g}}\right)
$$

where the approximate forms are valid if $k_{m, u, u}, k_{m, v, v} \ll k_{g}$ and then, the frequency difference is equal to

$$
\omega_{u}-\omega_{v} \approx \omega \frac{k_{m, u, u}-k_{m, v, v}}{2 k_{g}}
$$

\subsection{Motion of the pendulum}

In the general case, the excitation of the pendulum produces an oscillation which is a superposition of the two normal modes with amplitudes $u_{0}$ and $v_{0}$ and initial phases $\varphi_{u}$ and $\varphi_{v}$

$$
\begin{aligned}
& u(t) \quad=u_{0} \cos \psi_{u}(t) \text { and } v(t)=v_{0} \cos \psi_{v}(t) \\
& \text { with } \psi_{u}(t)=\omega_{u} t+\varphi_{u} \text { and } \psi_{v}(t)=\omega_{v} t+\varphi_{v}
\end{aligned}
$$

If the $\mathbf{x}, \mathbf{y}$ axes are deduced from the $\mathbf{u}, \mathbf{v}$ axes by a rotation of angle $\theta$, the pendulum position is given by

$$
\left[\begin{array}{c}
\epsilon_{x}(t) \\
\epsilon_{y}(t)
\end{array}\right]=\left[\begin{array}{cc}
\cos \theta & \sin \theta \\
-\sin \theta & \cos \theta
\end{array}\right]\left[\begin{array}{l}
u(t) \\
v(t)
\end{array}\right] .
$$

We note $x_{u}=u_{0} \cos \theta, x_{v}=v_{0} \sin \theta, y_{u}=-u_{0} \sin \theta$ and $y_{v}=v_{0} \cos \theta$. We thus get

$$
\begin{aligned}
\epsilon_{x}(t)= & {\left[x_{u}+x_{v}\right] \cos \psi_{-} \cos \psi_{+} } \\
& +\left[-x_{u}+x_{v}\right] \sin \psi_{-} \sin \psi_{+} \\
\epsilon_{y}(t)= & {\left[y_{u}+y_{v}\right] \cos \psi_{-} \cos \psi_{+} } \\
& +\left[-y_{u}+y_{v}\right] \sin \psi_{-} \sin \psi_{+}
\end{aligned}
$$

with $\psi_{+}(t)=\left[\psi_{u}(t)+\psi_{v}(t)\right] / 2$ and $\psi_{-}(t)=\left[\psi_{u}(t)-\psi_{v}(t)\right] / 2 . \psi_{+}(t)$ describes a fast oscillation at the normal mode mean frequency $\omega_{+}=\left(\omega_{u}+\omega_{v}\right) / 2$ and $\psi_{-}(t)$ a slow oscillation at the difference frequency $\omega_{-}=\left(\omega_{u}-\omega_{v}\right) / 2$. The modulus of the amplitudes of the fast oscillation are given by

$$
\begin{aligned}
& {\tilde{\epsilon_{x \omega_{+}}}}(t)=\sqrt{x_{u}^{2}+x_{v}^{2}+2 x_{u} x_{v} \cos \left(2 \psi_{-}(t)\right)} \exp (-t / \tau), \\
& {\tilde{\epsilon_{\omega_{+}}}}(t)=\sqrt{y_{u}^{2}+y_{v}^{2}+2 y_{u} y_{v} \cos \left(2 \psi_{-}(t)\right)} \exp (-t / \tau) .
\end{aligned}
$$

We have taken into account the damping of the oscillation by the factor $\exp (-t / \tau)$. We assume that the damping forces are equal for the two normal modes, as a consequence of the cylindrical symmetry of the pendulum (a possible exception may occur if the pendulum magnet induces eddy currents in nearby conductors). The amplitudes $\tilde{\epsilon_{x \omega_{+}}}(t)$ and $\tilde{\epsilon}_{\omega_{+}}(t)$ present beats at the frequency

$$
\omega_{\text {beat }}=\left|\omega_{u}-\omega_{v}\right| \approx \omega \frac{\left|k_{m, u, u}-k_{m, v, v}\right|}{2 k_{g}}
$$


The total (kinetic and potential) energy $E(t)$ is proportional to

$$
E(t)=\frac{\left(k_{g}+k_{m, u, u}\right) u_{0}^{2}+\left(k_{g}+k_{m, v, v}\right) v_{0}^{2}}{2} \exp (-2 t / \tau) .
$$

$E(t)$ decays because of damping but without any oscillation. The energy is partly transferred between the oscillations along the $\mathbf{x}$ and $\mathbf{y}$-directions but the total energy is conserved, if damping can be neglected. The energy transfer is complete in the particular case $u_{0}= \pm v_{0}$ and $\theta= \pm \pi / 4$.

\section{Toward an experiment}

We consider that the inhomogeneous part of the magnetic field $\mathbf{B}$ is produced by a magnetic dipole and that we can neglect the inhomogeneous part of the magnetic field due to other sources.

\subsection{Calculation of the magnetic potential energy}

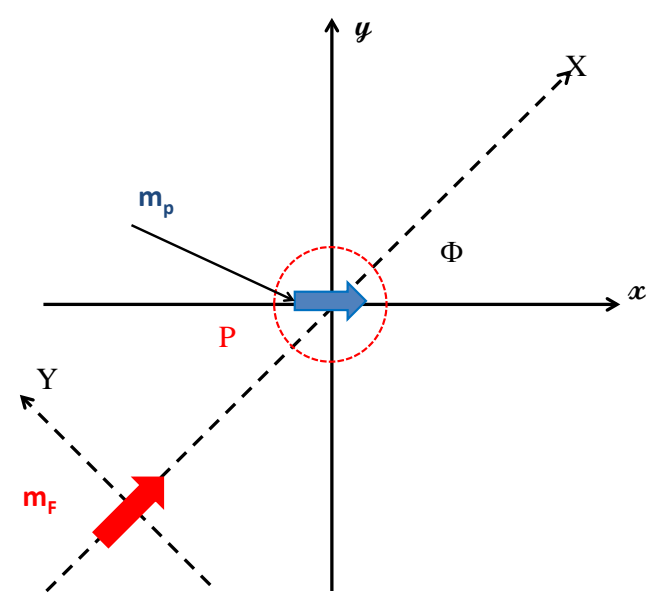

Figure 1. Schematic drawing of a top-view of the arrangement. The pendulum equilibrium position is at the origin of the $\mathbf{x}, \mathbf{y}$ system of axes while the magnetic moment $\mathbf{m}_{F}$, represented by a red arrow, is at the origin of the $\mathbf{X}, \mathbf{Y}$ system of axes. $\Phi$ is the angle between $\mathbf{X}$ and $\mathbf{x}$. The pendulum, represented by the red dashed circle, carries the magnetic moment $\mathbf{m}_{P}$ parallel to the $\mathbf{x}$-axis and represented by the blue arrow. The distance between the two magnetic moments is noted $r$.

Figure 1 represents the geometrical arrangement of our experiments. The magnetic moment $\mathbf{m}_{P}$ carried by the pendulum and the fixed magnetic moment $\mathbf{m}_{F}$ are in the same horizontal plane. The line going through the center of the fixed magnet and through the equilibrium position $O$ of the pendulum is parallel to $\mathbf{m}_{F}$. This line is chosen as the $\mathbf{X}$-axis of a second system of axes with its origin at the center of the magnetic moment $\mathbf{m}_{F}$. The $\mathbf{Y}$-axis is in the horizontal plane, perpendicular to the $\mathbf{X}$ axis, and the $\mathbf{Z}$-axis is in the vertical direction. We note $\Phi$ the angle between the $\mathbf{x}$ axis 
and the $\mathbf{X}$ axis. The potential energy of the magnetic moment $\mathbf{m}_{P}$ is given by

$$
E_{m}=-m_{P}\left(B_{X} \cos \Phi+B_{Y} \sin \Phi\right)
$$

The values of $B_{X}$ and $B_{Y}$, their first and second derivatives with respect to $X$ and $Y$ are given in the Appendix. From now on, we assume that the displacement of the equilibrium position of the pendulum due to the presence of the magnetic moment $\mathbf{m}_{F}$ is very small with respect to the distance $r$ between the two magnetic moments and we calculate the field derivatives at the equilibrium position in the absence of $\mathbf{m}_{F}$. Using eq. (6) written in the $X, Y$ coordinate system, we deduce the new equilibrium position

$$
\begin{aligned}
\frac{X_{e}-r}{r} & =-\frac{k_{m}}{4 k_{g}} \cos \Phi \text { and } \frac{Y_{e}}{r}=+\frac{k_{m}}{8 k_{g}} \sin \Phi \\
\text { with } k_{m} & =\frac{24 \mu_{0} m_{P} m_{F}}{4 \pi r^{5}}
\end{aligned}
$$

$k_{m}$ is a restoring force constant. We also rewrite eq. (7) in the $X, Y$ coordinate system

$$
\begin{aligned}
& E_{\text {pot }}\left(X_{e}+\varepsilon_{X}, Y_{e}+\varepsilon_{Y}\right)=\frac{k_{g}}{2}\left(\varepsilon_{X}^{2}+\varepsilon_{Y}^{2}\right) \\
& +\frac{k_{m}}{2}\left[-\varepsilon_{X}^{2} \cos \Phi+\varepsilon_{X} \varepsilon_{Y} \sin \Phi+\frac{\varepsilon_{Y}^{2}}{2} \cos \Phi\right]
\end{aligned}
$$

where the potential energy $E_{\text {pot }}\left(X_{e}, Y_{e}\right)$ has been omitted. To calculate the normal modes, we must give a diagonal form to the potential energy term. This calculation is easy but the general expressions are not very simple and we discuss only two particular cases in the next subsection.

\subsection{Two particular positions of the magnet $\mathbf{m}_{F}$}

In the case $\Phi=0$, the potential energy $E_{p o t}$ is given by

$$
E_{p o t}=\frac{1}{2}\left[k_{g}\left(\varepsilon_{X}^{2}+\varepsilon_{Y}^{2}\right)+k_{m}\left(-\varepsilon_{X}^{2}+\frac{\varepsilon_{Y}^{2}}{2}\right)\right]
$$

The matrix is already diagonal in the $X, Y$ basis and the normal modes correspond to oscillations along the $X$ and $Y$ directions with the angular frequencies

$$
\omega_{X}=\sqrt{\frac{k_{g}-k_{m}}{M}} \text { and } \omega_{Y}=\sqrt{\frac{k_{g}+\left(k_{m} / 2\right)}{M}}
$$

The beat frequency is given by

$$
\omega_{\text {beat }}=\left|\omega_{X}-\omega_{Y}\right| \approx \omega \frac{3 k_{m}}{4 k_{g}} .
$$

In the case $\Phi=\pi / 2$, the potential energy $E_{p o t}$ is given by

$$
E_{\text {pot }}=\frac{1}{2}\left[k_{g}\left(\varepsilon_{X}^{2}+\varepsilon_{Y}^{2}\right)+k_{m} \varepsilon_{X} \varepsilon_{Y}\right]
$$


The symmetry by exchange of $X$ and $Y$ proves that the potential is diagonal in the $U$, $V$ basis with $U=(X+Y) / \sqrt{2}$ and $V=(-X+Y) / \sqrt{2}$ i.e. in a basis rotated by an angle equal to $\pi / 4$. In this basis, $E_{\text {pot }}$ is given by

$$
E_{p o t}=\frac{1}{2}\left[k_{g}\left(\varepsilon_{U}^{2}+\varepsilon_{V}^{2}\right)+\frac{k_{m}}{2}\left(\varepsilon_{U}^{2}-\varepsilon_{V}^{2}\right)\right] .
$$

The normal modes correspond to the oscillations along the $U$ and $V$ axes with the angular frequencies

$$
\omega_{U}=\sqrt{\frac{k_{g}+\left(k_{m} / 2\right)}{M}} \text { and } \omega_{V}=\sqrt{\frac{k_{g}-\left(k_{m} / 2\right)}{M}}
$$

and the beat frequency is given by

$$
\omega_{\text {beat }}=\left|\omega_{V}-\omega_{U}\right| \approx \omega \frac{k_{m}}{2 k_{g}} .
$$

\section{The experiment}

\subsection{The set-up}

The pendulum is placed in a box to protect it from air currents. Its suspension wire is a piano string (diameter $0.49 \pm 0.01 \mathrm{~mm}$, length $404 \pm 0.5 \mathrm{~mm}$ ) which is rigid against rotation, so that the magnet rotation due to the torque exerted by the magnetic field is negligible and the angle $\Phi$ is constant. This pendulum was used to test the Stokes drag force on a sphere [9]. It is made of a plastic sphere (radius $a \approx 15 \mathrm{~mm}$ ) containing an aluminium alloy cylinder holding the pendulum magnet which is fixed to the piano string. Because the piano string wire is also rigid with respect to flexion, the restoring force is partly due to elastic forces. We have published the theoretical description of this type of pendulum [10]. We note $k_{\text {eff }}=M \omega^{2}$ the effective restoring force constant which replaces $k_{g}$. The fixed magnet is cubic (side length $20 \mathrm{~mm}$ ) and the pendulum magnet is a parallelepiped $\left(2 \times 3 \times 10 \mathrm{~mm}^{3}\right)$ : both magnets are made of various grades of sintered NdFeB alloys. Following Petruska and Abbott [12], for a cube-shaped magnet with uniform magnetization, the magnetic field of a cube-shaped magnet is very close to the field of a magnetic dipole even at distances as small as twice the cube edge length. We have measured this field as a function of the distance along the direction of the magnetic moment and we have thus deduced the magnetic moments $m_{P}$ and $m_{F}$ : their measured values agree with the value calculated with the equation $B_{r} V_{m} / \mu_{0}\left(V_{m}\right.$ being the magnet volume), using a remanent field $B_{r} \approx 1.3 \mathrm{~T}$, very close to the value given by the company which produced the magnets.

The pendulum oscillation is resonantly excited by the force exerted by a magnetic gradient on the pendulum magnet. To produce this magnetic field gradient, we use a flat coil with about 1000 turns and a mean radius $R \approx 15 \mathrm{~mm}$, located out of the box enclosing the pendulum, at a distance equal to $\sim 160 \mathrm{~mm}$ from the pendulum magnet. This coil is powered by a sine signal at the pendulum frequency. This signal 
is produced by the computer and amplified by a power amplifier. With a peak voltage equal to $6 \mathrm{~V}$ applied for about 20 seconds, we obtain oscillations amplitudes $\sim 0.5 \mathrm{~mm}$. The parameters (diameter, number of turns) of this coil have not been optimized: in particular, the maximum gradient force of a flat coil occurs at a distance equal to half its radius and a considerably more efficient excitation is obviously possible.

To measure simultaneously the two displacements $\varepsilon_{x}(t)$ and $\varepsilon_{y}(t)$ of the pendulum, we use two shadow detectors described in our previous paper [9]. With a laser beam radius $w \approx 1 \mathrm{~mm}$, the sensitivity is about $1-2 \times 10^{3} \mathrm{~V} / \mathrm{m}$ and the deviations from linearity are below $5 \%$ for a displacement smaller than $\pm 0.5 \mathrm{~mm}$, as measured using the shadow of a knife edge placed on a translation stage. The shadow detector signals are digitized at $40 \mathrm{~Hz}$ and we thus get the measurements of the displacements $\epsilon_{x}(t)$ and $\epsilon_{y}(t)$ as a function of the time $t$.

In another set of experiments, we have used a magnetic induction detector made of a pair of similar coils of solenoid valves [11]: this detector is less sensitive than the shadow detectors. We compensate this reduced sensitivity by increasing the pendulum magnet volume to $1 \mathrm{~cm}^{3}$ and the oscillation amplitude to a few millimeters. We thus obtain very nice signals. We have then used a $1 \mathrm{~cm}^{3}$ magnet as the fixed magnet so that the magnetic moment product $m_{P} m_{F}$ keeps a similar value. The advantages of the magnetic induction detector are that it does not require any fine alignment and that its linearity range is considerably larger. However, as we have only one such detector, we have recorded only one displacement, $\epsilon_{x}(t)$ : this explains why we do not present the results of this second set of experiments. In order to record the two displacements with two such detectors, the coils should be placed on the axes $(\mathbf{x} \pm \mathbf{y}) / \sqrt{2}$.

\subsection{The experiments}

With the excitation coil axis and the pendulum magnet aligned with the $\mathbf{x}$-axis, we excite the oscillation in this direction. We have fixed the angle $\Phi=\pi / 2$ because, in this case, the normal modes are theoretically excited with the same amplitude, leading to the

maximum beat contrast. Moreover, when $\Phi=\pi / 2$, the displacement of the pendulum is along the $\mathbf{Y}$-axis (see eqs; 19) and the modification of the distance $r$, being of the

Table 1. This table gives the mass $M$ of the pendulum body, its oscillation frequency $\omega /(2 \pi)$, the effective restoring force constant $k_{e f f}=M \omega^{2}$, the dimensions and magnetic moments $m_{P}$ and $m_{P}$ of the two magnets.

\begin{tabular}{|c|c|}
\hline $\mathrm{M}(\mathrm{g})$ & 20.9 \\
\hline$\omega /(2 \pi)(\mathrm{Hz})$ & 0.844 \\
\hline$k_{\text {eff }}(\mathrm{N} / \mathrm{m})$ & 0.588 \\
\hline$m_{P}\left(\mathrm{~A} . \mathrm{m}^{2}\right)$ & $0.060 \pm 0.003$ \\
\hline$m_{F}\left(\mathrm{~A} . \mathrm{m}^{2}\right)$ & $8.5 \pm 0.2$ \\
\hline
\end{tabular}


second order in the displacement, is negligible. We have measured the beat pattern for various values of the distance $r$ between the centers of the two magnets.

\subsection{Experimental protocol}

We first measure the shadow detector signal during $200 \mathrm{~s}$ with a numerical oscilloscope and, using its FFT function, we measure the two pendulum frequencies, if they are resolved or their mean value in the opposite case. Then, we choose the excitation frequency as the mean of the two frequencies in order to excite equally the two normal modes. After the excitation period, the pendulum oscillates freely for a duration equal to $4000 \mathrm{~s}$ before a new excitation. Because of the sensitivity of the shadow detectors, any adjustment of pendulum or of the shadow detectors put the pendulum in oscillation and the damping of this oscillation takes a long time. Because of this uncontrolled excitation, the first excitation period usually occurs while the pendulum is already oscillating and the initial conditions are ill-defined while at the end of a recording period, the oscillation amplitudes are negligibly small and the excitation puts the pendulum in a well-defined state. We show in figure 2 an example of a small part of the recorded signals. The beat patterns are obvious and they exhibit a very good contrast.

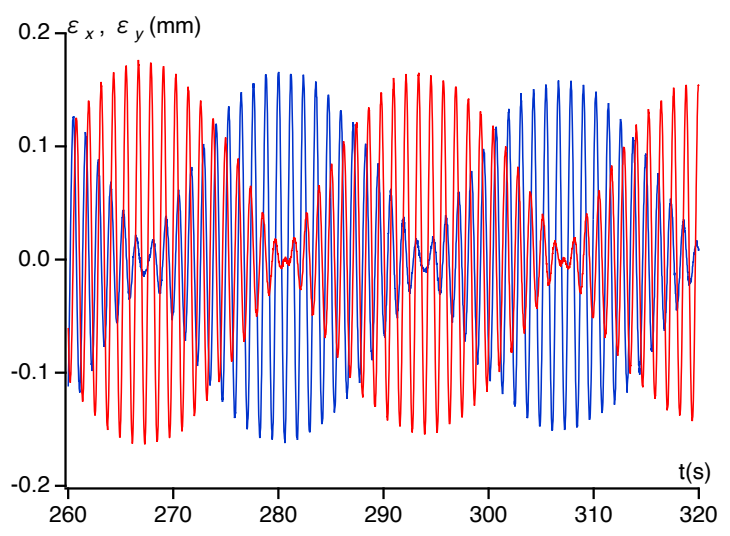

Figure 2. The recorded signals $\epsilon_{x}(t)$ (blue line) and $\epsilon_{y}(t)$ (red line) converted in millimeters, thanks to the measured sensitivities of the shadow detectors. The experiment is done with a distance $r=140 \pm 2 \mathrm{~mm}$ and the beat period is $T_{\text {beat }}=$ $26.47 \pm 0.02 \mathrm{~s}$.

\subsection{Data analysis}

We extract the oscillation amplitudes of the fast oscillation $\tilde{\epsilon_{x \omega_{+}}}(t)$ and ${\tilde{\epsilon_{\omega_{+}}}}(t)$ by a sliding Fast Fourier Transform over 256 points i.e. over 6.4 s. The measured amplitudes are fitted by the following equation

$$
\tilde{\epsilon}_{\omega_{+}}(t)=\sqrt{A+B \cos \left(\frac{2 \pi t}{T_{\text {beat }}}+\phi\right)} \exp (-t / \tau),
$$


which is equivalent to eq. (15). Figure 3 presents an example of these amplitudes and the squared amplitudes with their fits by eq. (28). The quantities $A_{i}$ and $B_{i}(i=x$ or $i=y$ ) would be equal in the ideal case and the beat contrast is then maximum. However, any misalignment of the magnetic moments $m_{P}$ and $m_{F}$ and of the direction of the excitation modifies the relative amplitudes of these modes. Because of the long duration of the experiments, we have not made much effort to improve the beat contrast.
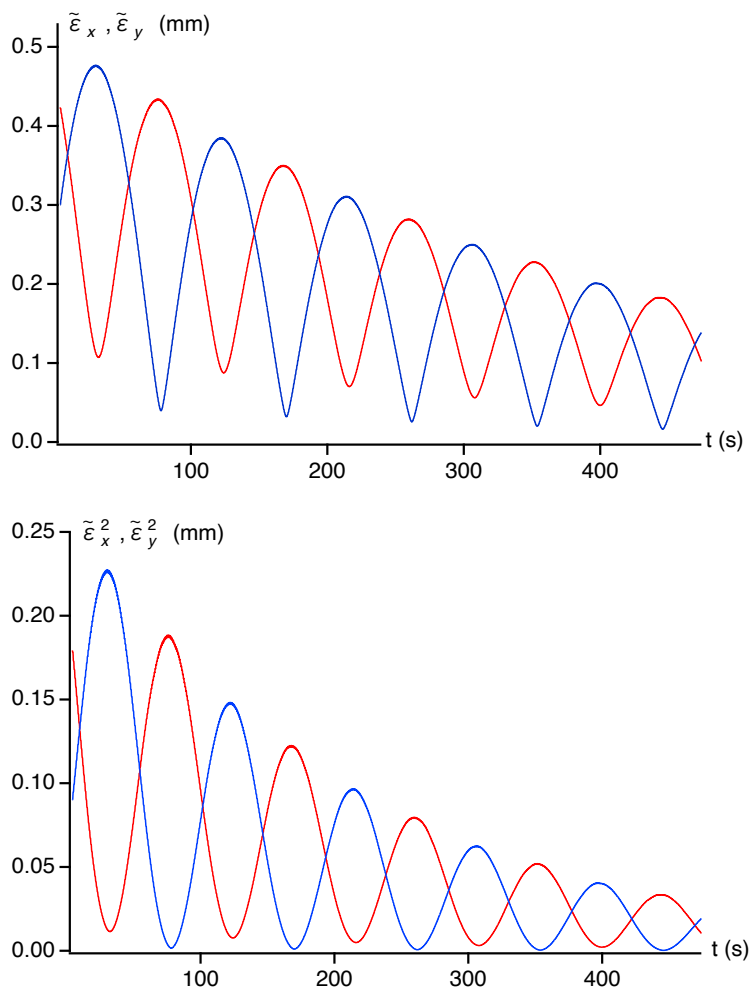

Figure 3. The amplitudes of the fast oscillations converted in millimeters thanks to the measured sensitivities of the shadow detectors. Upper panel: the amplitudes $\tilde{\epsilon}_{x, \omega_{+}}(t)$ (blue full line) and $\tilde{\epsilon}_{y, \omega_{+}}(t)$ (red full line). Lower panel : the squares of these amplitudes showing the almost complete energy exchange between the oscillation along the $\mathbf{x}$ and $\mathbf{y}$ directions. The experiment is done with a distance $r=180 \pm 2 \mathrm{~mm}$ leading to a beat period $T_{\text {beat }}=91.94 \pm 0.04 \mathrm{~s}$.

We have measured the beat period $T_{\text {beat }}$ for various values of the distance $r$ between the magnet centers, covering the $140-220 \mathrm{~mm}$ range by $20 \mathrm{~mm}$ steps. Equation (27) relates this period to the pendulum period $T_{\text {pend }}=2 \pi / \omega$ by

$$
\begin{aligned}
\frac{T_{\text {beat }}}{T_{\text {pend }}} & =\frac{2 k_{\text {eff }}}{k_{m}}=C r^{5} \\
\text { with } C & =\frac{4 \pi k_{e f f}}{12 \mu_{0} m_{P} m_{F}}
\end{aligned}
$$

where the dependence in $r^{5}$ is deduced from the dependence of $k_{m}$ given by equation (19). This behavior is tested in fig. 4. The best fit to the measured beat period gives 
$C=(4.17 \pm 0.012) \times 10^{5} \mathrm{~m}^{-5}$ while the value of $C$ deduced from the measured magnetic moments is $C=(4.8 \pm 0.4) 10^{5} \mathrm{~m}^{-5}$.

With $\Phi=\pi / 2$, we deduce from eq. (19) that $X_{e}=0$ and

$$
\frac{Y_{e}}{r}=\frac{k_{m}}{8 k_{\text {eff }}}=\frac{T_{\text {pend }}}{8 T_{\text {beat }}} .
$$

In our experiments, when $r$ increases from 140 to $220 \mathrm{~mm}$, the ratio $T_{\text {pend }} / T_{\text {beat }}$ varies from about 0.045 down to 0.0047 and the ratio $Y_{e} / r$ decreases from $1.1 \%$ to $0.12 \%$ and the modification of the distance due to this displacement is fully negligible.

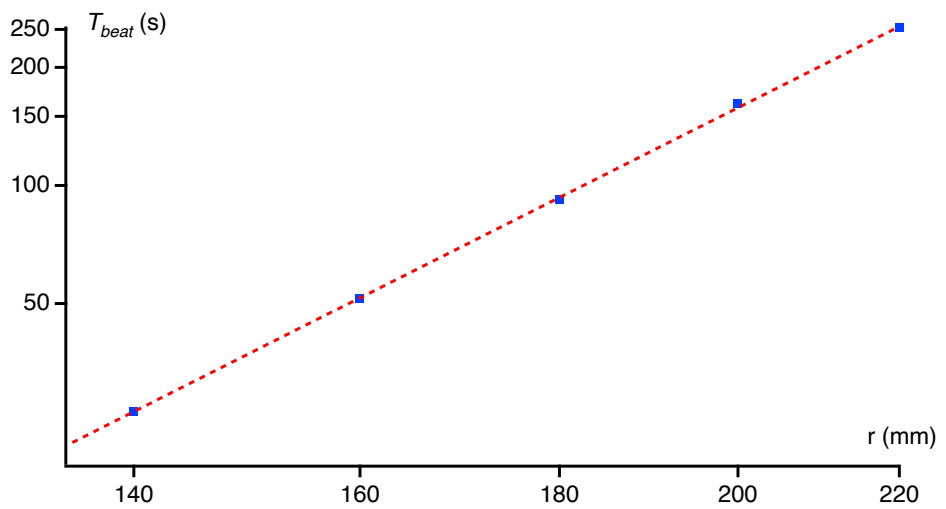

Figure 4. The beat period $T_{\text {beat }}(\mathrm{s})$ is plotted as a function of the distance $r(\mathrm{~mm})$ between the centers of the two magnets in a loglog plot. The squares represent the experimental results and the dashed straight line is the best fit following $T_{\text {beat }} \propto r^{5}$.

\subsection{Direct observation of the pendulum motion}

If the pendulum starts oscillating along the $\mathrm{x}$-axis at $t=0$, its oscillation becomes elliptic and at $t=T_{\text {beat }} / 4$, the oscillation is circular in one sense (depending of the sense of the two magnetic moment); then after a period of elliptic oscillation, the oscillation becomes linear along the $\mathbf{y}$-axis at $t=T_{\text {beat }} / 2$; at $t=3 T_{\text {beat }} / 4$, the oscillation is circular in the opposite sense and at $t=T_{\text {beat }}$, the oscillation is back to linear along the $\mathbf{x}$-axis exactly as in $t=0$. Figure 5 illustrates this behavior.

We have also made a movie of the pendulum motion in the configuration $\Phi=\pi / 2$, with an excitation along the $\mathbf{x}$-axis at $t=0$. The pendulum oscillation follows our theoretical predictions: some minor deviations are due to the large amplitude used in this experiment which makes that the pendulum explore a large spatial region while our calculation is valid in the small amplitude limit [13].

\section{Concluding remarks}

We have studied the motion of a pendulum having the cylindrical symmetry, with two normal modes which are degenerate. We have shown that, if the pendulum 


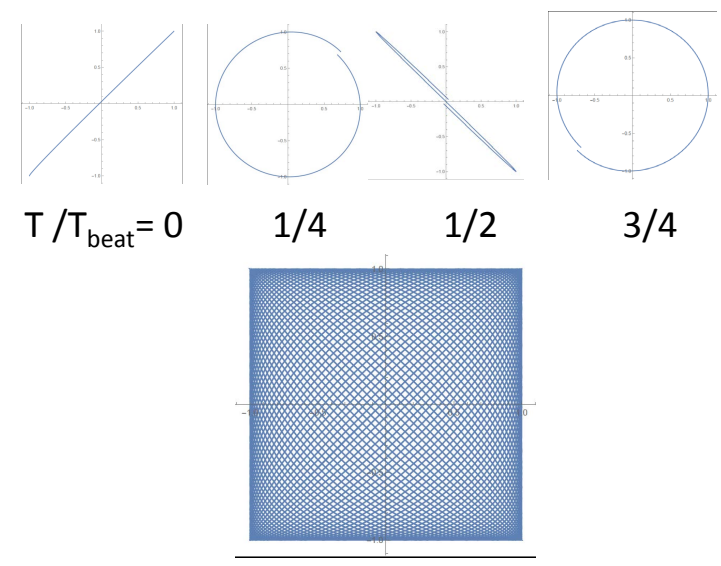

Figure 5. Plot of the calculated motion of the pendulum in the $u, v$ plane, assuming an equal excitation of the two normal modes in the case $T_{\text {beat }}=100 T_{\text {pend }}$. Each top panel describes the motion during a time interval equal to the pendulum period, chosen at multiples of $T_{\text {beat }} / 4$. The bottom panel describes the motion during one full beat period $T_{\text {beat }}$, exhibiting a Lissajous curve.

carries a magnetic moment, an inhomogeneous magnetic field displaces the equilibrium position and lifts the mode degeneracy. The resulting normal modes are defined by the symmetry of the perturbation. In the present case, the perturbation is conserved by time reversal, because, although a magnetic dipole changes sign by time-reversal, the perturbation involves the product of two magnetic dipoles: the normal modes correspond to linear oscillations. The Foucault's pendulum is the most famous case in which the perturbation, due to Coriolis effect, is not conserved by time reversal and, in this case, the normal modes are circular oscillations.

We have shown that, when the mode degeneracy is lifted, the pendulum oscillation measured along a given direction exhibit beats and, with a correct choice of parameters, the energy transfer from one direction of oscillation to the perpendicular direction can be complete in theory and almost complete in our experiments. We have also verified that the beat period varies as $r^{5}$, where $r$ is the distance between the magnet centers, in good agreement with theory.

\section{Acknowledgments}

We want to thank D. Castex, E. Panader, S. Faure, L. Polizzi and W. Volondat for their contributions to the experiment. Financial supports from CNRS INP, CNRS MI DEFI and université P. Sabatier are gratefully acknowledged.

\section{Appendix I: Magnetic field of a permanent magnet}

The magnetic field produced by a magnet is given by its multipolar expansion and, because $\nabla \cdot \mathbf{B}=0$, one can prove that this expansion contains only odd power terms 
[12]. The field due to the $2^{2 n+1}$-pole decreases like $r^{-(2 n+1)}$ where $r$ is the distance to the magnet center: the first non-vanishing terms is the dipolar term with $\mathbf{B} \propto r^{-3}$ and for the next non-vanishing term $\mathbf{B} \propto r^{-5}$. If $r$ is large with respect to the magnet dimensions, the magnetic field $\mathbf{B}$ of a compact magnet with a uniform magnetization is very well approximated by the dipolar term [12] and the magnetic moment $\mathbf{m}$ is equal to

$$
\mu_{0} \mathbf{m}=V_{m} \mathbf{B}_{\mathbf{r}}
$$

where $V_{m}$ is the magnet volume and $\mathbf{B}_{r}$ is the remanent field of the magnet.

The magnetic field of a magnetic moment $\mathbf{m}$ are usually expressed in polar coordinates. Here, we write the components of the magnetic field in a cartesian coordinate system $\mathbf{X}, \mathbf{Y}$ and $\mathbf{Z}$, with the $\mathbf{X}$-axis parallel to the magnetic moment $\mathbf{m}$ and with the origin at the center of the magnet

$$
\begin{aligned}
& B_{X}=\frac{\mu_{0} m_{F}}{4 \pi} \times \frac{2 X^{2}-Y^{2}-Z^{2}}{\left(X^{2}+Y^{2}+Z^{2}\right)^{5 / 2}} \\
& B_{Y}=\frac{\mu_{0} m_{F}}{4 \pi} \times \frac{3 X Y}{\left(X^{2}+Y^{2}+Z^{2}\right)^{5 / 2}} \\
& B_{Z}=\frac{\mu_{0} m_{F}}{4 \pi} \times \frac{3 X Z}{\left(X^{2}+Y^{2}+Z^{2}\right)^{5 / 2}}
\end{aligned}
$$

We need the first and second derivatives of $B_{X}$ and $B_{Y}$ with respect to $X$ and $Y$ for $Z=0$. Their calculation is simple but tedious and we have used Mathematica to verify our results. The first derivatives are equal to

$$
\begin{aligned}
\frac{\partial B_{X}}{\partial X} & =\frac{\mu_{0} m_{F}}{4 \pi} \times \frac{3 X\left(-2 X^{2}+3 Y^{2}\right)}{\left(X^{2}+Y^{2}\right)^{7 / 2}} \\
\frac{\partial B_{X}}{\partial Y} & =\frac{\partial B_{Y}}{\partial X}=\frac{\mu_{0} m_{F}}{4 \pi} \times \frac{3 Y\left(-4 X^{2}+Y^{2}\right)}{\left(X^{2}+Y^{2}\right)^{7 / 2}} \\
\frac{\partial B_{Y}}{\partial Y} & =\frac{\mu_{0} m_{F}}{4 \pi} \times \frac{3 X\left(X^{2}-4 Y^{2}\right)}{\left(X^{2}+Y^{2}\right)^{7 / 2}}
\end{aligned}
$$

At the pendulum equilibrium position in the absence of the magnet, $X=r$ with $r>0$, $Y=0, Z=0$, the first derivatives of $B_{X}$ and $B_{Y}$ are given by

$$
\begin{aligned}
& \frac{\partial B_{X}}{\partial X}=-\frac{6 \mu_{0} m_{F}}{4 \pi r^{4}}, \quad \frac{\partial B_{X}}{\partial Y}=0 . \\
& \frac{\partial B_{Y}}{\partial X}=0, \quad \frac{\partial B_{Y}}{\partial Y}=\frac{3 \mu_{0} m_{F}}{4 \pi r^{4}}
\end{aligned}
$$

The second derivatives are equal to

$$
\frac{\partial^{2} B_{X}}{\partial X^{2}}=\frac{\mu_{0} m_{F}}{4 \pi} \times \frac{3\left(8 X^{4}-24 X^{2} Y^{2}+3 Y^{4}\right)}{\left(X^{2}+Y^{2}\right)^{9 / 2}}
$$




$$
\begin{aligned}
\frac{\partial^{2} B_{X}}{\partial X \partial Y} & =\frac{\mu_{0} m_{F}}{4 \pi} \times \frac{15 X Y\left(4 X^{2}-3 Y^{2}\right)}{\left(X^{2}+Y^{2}\right)^{7 / 2}}=\frac{\partial^{2} B_{Y}}{\partial X^{2}} \\
\frac{\partial^{2} B_{X}}{\partial Y^{2}} & =\frac{\mu_{0} m_{F}}{4 \pi} \times \frac{3\left(-4 X^{4}+27 X^{2} Y^{2}-4 Y^{4}\right)}{\left(X^{2}+Y^{2}\right)^{9 / 2}}=\frac{\partial^{2} B_{Y}}{\partial X \partial Y} \\
\frac{\partial^{2} B_{Y}}{\partial Y^{2}} & =\frac{\mu_{0} m_{F}}{4 \pi} \times \frac{15 X Y\left(-3 X^{2}+4 Y^{2}\right)}{\left(X^{2}+Y^{2}\right)^{9 / 2}}
\end{aligned}
$$

The presence of equalities among these derivatives is not surprising. As $\Delta \mathbf{B}=0$ and $\nabla \times \mathbf{B}=0$ in free space, one can prove that the 27 second derivatives $\partial^{2} B_{i} / \partial X_{j} \partial X_{k}$ can be expressed as a functions of 7 of them only. At the equilibrium position in the absence of the magnet, $X=r, Y=0, Z=0$, the second derivatives of $B_{X}$ and $B_{Y}$ are given by

$$
\begin{aligned}
\frac{\partial^{2} B_{X}}{\partial X^{2}} & =\frac{24 \mu_{0} m_{F}}{4 \pi r^{5}}, & & \frac{\partial^{2} B_{X}}{\partial X \partial Y}=0, \\
\frac{\partial^{2} B_{X}}{\partial Y^{2}} & =-\frac{12 \mu_{0} m_{F}}{4 \pi r^{5}}, & & \frac{\partial^{2} B_{Y}}{\partial X^{2}}=0, \\
\frac{\partial^{2} B_{Y}}{\partial X \partial Y} & =-\frac{12 \mu_{0} m_{F}}{4 \pi r^{5}}, & & \frac{\partial^{2} B_{Y}}{\partial Y^{2}}=0 .
\end{aligned}
$$

[1] G.L. Baker and J.A. Blackburn, The pendulum. A case study in physics (Oxford University Press, 2005).

[2] The pendulum: Scientific, Historical, Philosophical and Educational Perspectives edited by M.R. Matthews, C.F. Gauld, A. Stinner, (Springer, 2005).

[3] L.P. Pook, Understanding Pendulums. A Brief Introduction, History of Mechanism and Machine Science, Volume 12, Series Editor M.G. CECCARELLI (Springer Springer Dordrecht Heidelberg London New York 2011).

[4] A.D. Crowell, "Motion of the Earth as viewed from the moon and the Y-suspended pendulum," Am. J. Phys. 49, 452-454 (1981).

[5] R.J. Whitaker, "A note on the Blackburn pendulum," Am. J. Phys. 59, 330 (1991).

[6] M.L. Foucault, " Démonstration physique du mouvement de rotation de la terre au moyen du pendule," C. R. Acad. Sci. D 32, 135 (1851).

[7] J.H. Hannay, "Angle variable holonomy in adiabatic excursion of an integrable Hamiltonian," J. Phys. A: Math. Gen. 18 221-230 (1985).

[8] J. von Bergmann and H.C. von Bergmann, "Foucault pendulum through basic geometry," Am. J. Phys. 75, 888 (2007).

[9] G. Dolfo, J. Vigué, and 'D. Lhuillier, Experimental test of unsteady Stokes' drag force on a sphere," Experiments in Fluids 61, 97 (2020).

[10] G. Dolfo and J. Vigué, "A more accurate theory of a flexible-beam pendulum", Am. J. Phys. 83, 525-530 (2015).

[11] G. Dolfo, D. Castex, and J. Vigué, "Damping mechanisms of a pendulum", Eur. J. Phys. 37, 065004 (2016).

[12] A.J. Petruska and J.J. Abbott, "Optimal Permanent-Magnet Geometries for Dipole Field Approximation," IEEE Trans. on Magnetics 49, 811-819 (2013).

[13] Supplementary file. 